\title{
Data and Knowledge for a Healthy Planet
}

\subsection{Data and Knowledge: State and Future}

Current science justifies policy action now, but more detailed knowledge can enable more refined and preemptive policy. Existing knowledge is already sufficient to mobilize action today. New knowledge, including disaggregated data from Earth Observation, in situ data, citizen science, socially-disaggregated data, ground-truthing and indigenous and local knowledge, needs to be brought into national policy and accounting more broadly. There are major benefits in accounting systems that register the details about who causes damage to the environment, how and why; what is the extent of nature's contributions to humans and the loss of ecosystem goods and services; and who is affected. Statistics and accounting systems also need to recognize the realities of predominantly poor people in the informal economy, who are often particularly dependent on nature's contributions to people and hence more vulnerable to environmental degradation. \{Co-Chairs' Message\}

\section{Figure 6.1: Global hydrological fluxes and storages (expressed in 1,000 $\mathrm{km}^{3}$ per year), illustrating natural and}

anthropogenic cycles

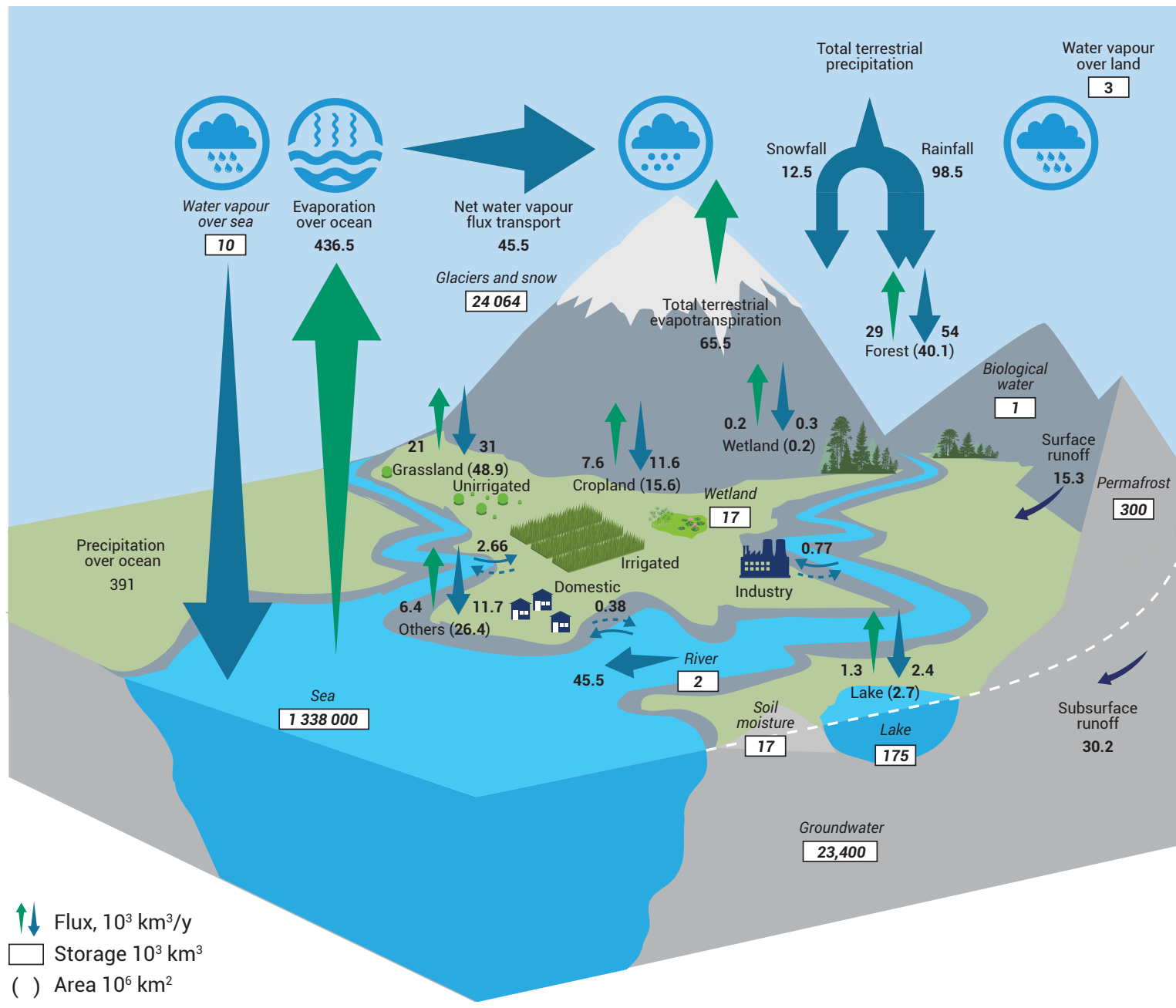

Source: Oki and Kanae (2006). 
Meeting environmental challenges will require advances in environmental indicators and analysis, particularly analysis that addresses interlinkages across different environmental domains and among the environment, society and the economy (well established). Recent advances in collecting official statistics related to the environment, including geospatial ones, contribute to environmental monitoring and permit representations of environmental flows (Figure 6.1). However, there are still methodological gaps in measuring many aspects of the environment; there is very limited information linking social and environmental processes; and there are significant capacity gaps in countries attempting to build their environmental information and natural capital accounting systems. \{Chapter 3, ExecSum\}

\section{Meeting the SDGs will require a new commitment} to environmental data collection. (established but incomplete) Global commitments to the SDGs increase global recognition that monitoring the environmental dimension of development requires regular, standardized data collection, including time series statistics and indicators. Monitoring progress on the SDGs requires shifts in data collection, analysis and dissemination, including the use of environmental statistics, geospatial data, earth observation and new data sources (i.e. citizen science, big data, traditional knowledge). The number of Tier 2 and Tier 3 environmental indicators relating to the SDGs (Figure 6.2 and Figure 6.3) shows that implementing the SDGs calls for a data revolution, incorporating

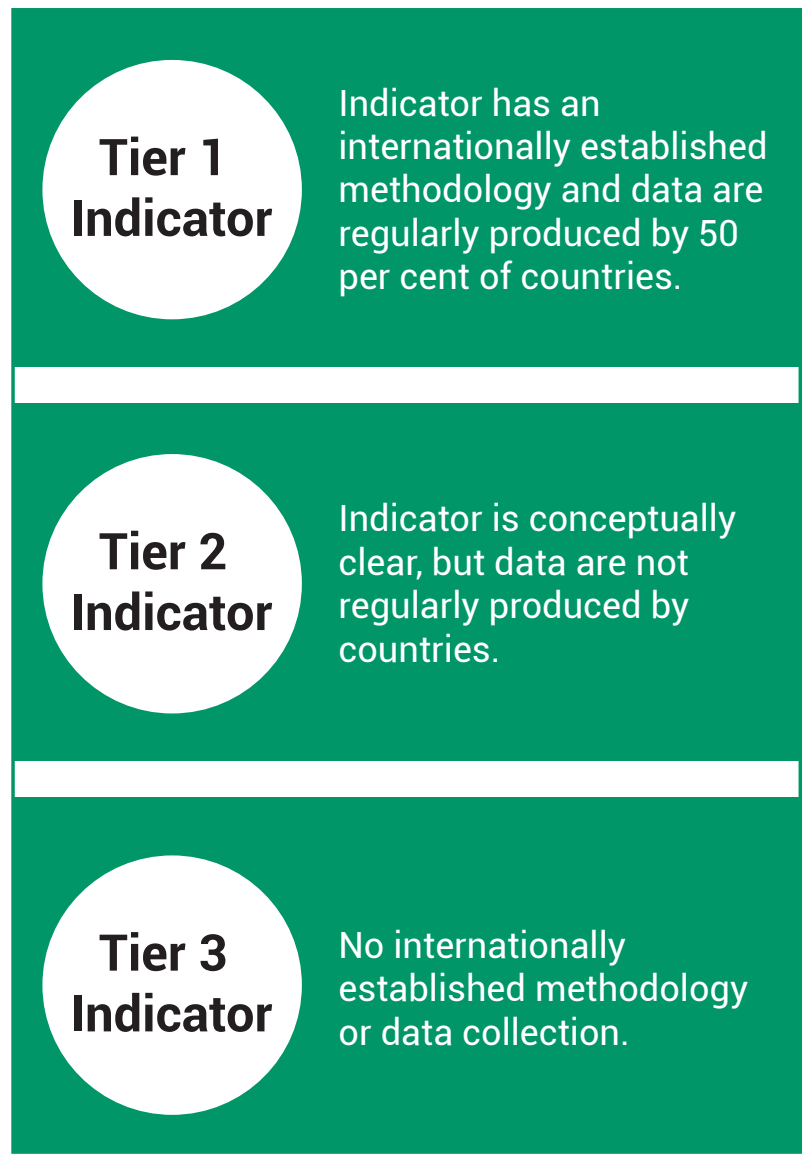

Source: United Nations (2018a).

\section{Figure 6.3: Environment-related SDG indicators by goal and tier}

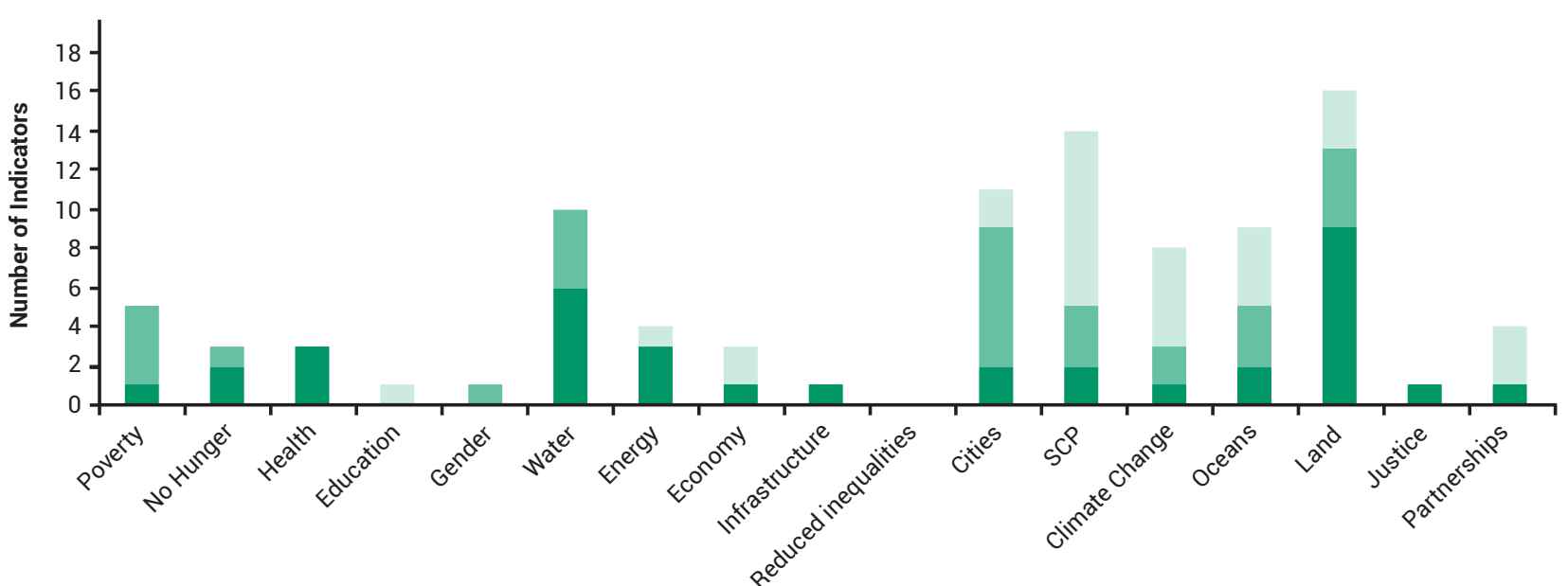

Sustainable Development Goals

Tier I

Source: United Nations (2018a). 
disaggregated data, to ensure that no one is left behind, and for reporting at all levels of the 17 goals. New and innovative approaches to data and knowledge systems, with an aligned focus on evidence-based information gathering, are essential for achieving the ambitious SDG framework. $\{25.4 .8\}$

However, monitoring the entire SDG framework over the 2016-2030 period is estimated to cost as much as a quarter of a trillion dollars. So, in addition to improving data systems, there is also a need for priority setting to target data collection and improve efficiencies. $\{3.8\}$

\section{Currently, most environmental indicators capture the geophysical characteristics of environmental states and processes (well established). Environmental processes are crucially influenced, however, by the interactions between physical and social systems. Without information - and data - on both domains and their interactions, the picture of the current situation and direction of travel will be incomplete. A combination of conventional methods with emerging forms of data and knowledge, including social data, provides a holistic view of the environment, encompassing social, physical and economic perspectives. \{25.4.8\} Environmental change is difficult to measure and its effects are even more complicated to measure, especially in relation to identifying causes. A shift from focusing solely on physical dimensions to including social orientation, economic value and impacts on health and well-being is crucial, although it is a challenge for even well- developed statistical systems $\{3.8\}$}

The usefulness of knowledge and data depends on what the data represents and the issues it addresses (well established). The ability to fund data ensures that data serves the needs of different communities, and assesses whose knowledge counts in critical in defining good quality policies. As highlighted in GEO-6, Chapter 3 , the nexus between society and the environment can only be assessed if there is disaggregated information on different populations since not everybody has the same level of dependence on the environment, nor does everybody have the same impact on it. Therefore, there is a need for information that can be disaggregated by income, gender, age, ethnicity, migratory status, disability, geographical location and other characteristics relevant in national contexts. Unfortunately, there is currently a dearth of environmentrelated information that can be disaggregated; data from household surveys on access to water, energy and other natural resources is available only at the household level, which makes understanding differences at other levels difficult. $\{25.4 .1\}$ Moreover, the question of which available data should be acted on, or what kind of information, based on which assumptions, and which reality counts, is almost entirely a problem that must be resolved in the social realm. $\{25.4 .8\}$

Advances in collecting official statistics and other evidence that feed into geographic information systems for environmental monitoring and accounting have expanded knowledge, while highlighting data gaps in every environmental domain (well established). Such gaps limit our capacity to formulate and implement policy solutions. Time series data are vitally important in this regard, as they form the basis for monitoring change. Regular standardized data collection can be translated into statistics and indicators that highlight vulnerabilities within and between communities. Disaggregated data that capture information by gender, ethnicity, race, income, age and geographic region can identify critical differences and promote effective policy design. $\{3.5,3.7$; SPM $\}$

\subsection{The Primary Domains of Data and Needs in those Domains}

There are growing amounts of data on the health impacts of environmental challenges, but these data are not always adequate for some world regions and are often not disaggregated by gender, class or social status (well established). Combined physical and social environments have strong influences, both direct and indirect, on human health and well-being. Measuring linkages between "exposure" and "outcome" and assessing causal relationships and the exposure of populations to environmental changes, requires strong statistical bases and large sample sizes. Effective largescale epidemiological studies require the development of data systems with high degrees of security and reliability. Many governments and institutions do not have the capacity to enact or even to effectively use the findings of such systems. Recent developments in big data may allow the assessment of long-term environmental exposures. However, establishing causal relationships between human exposure to environmental damage and subsequent health outcomes is complicated and contested. It is necessary to explore the use of multiple forms and sources of information, such as citizen science and traditional knowledge, to establish and validate the long-term effects of human activities and natural disturbance such as climate change on health. $\{3.8\}$

Measuring the nexus between gender and the environment has been identified as a high priority; women and men, in many contexts, have differing rights over and access to the environment (well established). Furthermore, women and men often have 
different perceptions of environmental problems; have different vulnerabilities to environmental degradation and hazards; and often play different roles in environmental management decision-making. Currently, only very limited time series data and statistics are available on the gender-environment nexus. \{Chapter 3, ExecSum, 3.5\} Producing gender statistics entails disaggregating data by sex (and other intersectional characteristics) to reveal differences or inequalities in environmental relationships. Collecting effective gender statistics also requires rethinking data collection norms and techniques in order to ensure that the diversity of various groups of women and men, and their specific activities and challenges, are captured; for example, data collected at "household" level masks intra-household gender differences in access to and use of resources. Gender-specific data inadequacies (e.g. persistent underreporting of women's economic activity, underreporting of violence against women, and undercounting of girls, their births and their death) must be addressed in order to develop a realistic understanding of social-environmental relations. $\{$ Box 3.2$\}$

\section{Citizen science is providing unprecedented} opportunities to engage the public in collecting and analyzing vast amounts of environmental data (well established). The potential of widely dispersed teams of observers, together with new technologies such as smart sensors, mobile telephony, the Internet and computing capabilities, is offering new approaches for research and for engaging the public on environmental issues. In addition to the collection of large amounts of data, the advancement of new technologies has enhanced the quality and veracity of the data collected. Key opportunities presented by citizen science include greater frequency of data from dispersed sources; the ability to address large knowledge and funding deficits; the ability to educate the public about environmental policy issues; and use of local knowledge. However, combining citizen science-derived information with other data systems (especially earth observation systems) presents significant data compatibility and comparability challenges. \{Chapter 25, ExecSum, 25.2.1\}

\section{Traditional knowledge is a globally underutilized resource which can complement science-based knowledge (well established). In 2007 the United Nations Declaration on the Rights of Indigenous Peoples helped peoples to document, revive and strengthen their knowledge. However, capacity- building is needed to develop practices for managing the collection of information and the integration of traditional knowledge with other knowledge systems.}

Collaborative work among traditional knowledge holders, academia and governments has led to innovative processes, procedures and tools for data generation, and for knowledge production and enrichment, which can help in understanding and caring for the environment. \{25.1.3; SPM $\}$

\section{Many environmental data do not systematically assess the economic costs of action and inaction} (well established). Existing economic-environmental data are scattered and fragmented; data on inactions are growing but inadequate. The economic evaluation of environmental impacts will need to include overall assessment of nature's contributions to people's lives; an accounting of the impacts of global economic activities, investment and trade on people and the environment; and the comprehensive institutional issues affecting equity and market operations. Specific findings on sustainability can only be obtained through robust analysis covering ecological, social and cultural factors and their interactions over time. Economic analysis of the environment should be oriented towards the wider scope of the SDGs, including peace, equity and security. Monetary and non-monetary values in relation to the environment and resources (as well as models reflecting the economics of nature) can only be generated through the use of timely and reliable knowledge and data from statistical surveys and other new sources such as big data, gender-disaggregated data, and information drawn from citizen science and indigenous knowledge. $\{3.8\}$

\section{Earth Observation is the primary contributor to} remotely sensed big data (well established). When coupled with emerging technologies such as smart sensors, mobile devices and web applications, citizen science and other forms of localized data collection, Earth Observation enables the collection and analysis of large volumes of geographically-referenced data to inform and support decision-making, educate the public about environmental issues, and enhance public participation $\{25.4 .1\}$.

\footnotetext{
Whether an indicator can be measured by Earth Observation is a major factor in data availability (well established). A revolution in the quality and costeffectiveness of Earth Observation data means that indicators that can be measured remotely have far greater spatial coverage than those which cannot. For example, deforestation and land use change can be measured with increasing accuracy using satellites, but satellites cannot monitor all aspects of subsurface ocean environments. Data are particularly sparse for biodiversity, which is measured mostly by in situ
} 
observation and genetic analysis. Some freshwater components, such as groundwater and water use, are also data-deficient due to measurement challenges. The dichotomy in the volume of remotely sensed data versus in situ data will inevitably grow as Earth Observation technologies improve. This should not lead to a bias in understanding, and should be compensated by resources for adequate ground-truthing and in situ data. $\{3.4$; SPM $\}$

Future sensor technology should allow detailed data disaggregation of spatial and demographic information (established but incomplete). A combination of satellites and airborne and ground-based networks can help monitor developments and impacts at the local, regional and global levels in near-real time. The resulting data and information, together with rapidly emerging digital infrastructure, can enable rapid response to changing circumstances. Realizing those benefits, however, depends on appropriate governance and national circumstances for data collection, processing, curation and use, along with combining environmental data with context-relevant socioeconomic information. \{25.1.2; SPM

There are inadequate data on indicators for equity and on human-environment interactions (well established). Collection, disaggregation and analysis of data for the most vulnerable communities and for indigenous communities remain a challenge. More work in this area would better capture issues of inequality. It is important to promote data and knowledge on how to overcome barriers to political and social participation that perpetuate discrimination and exclusion. In terms of the geographic distribution of environmental research, most is concentrated in the United States of America, China, Japan and Germany, which together account for 63 per cent of the global research and development expenditures, mostly funded by the business sector. Businesses as funders of research have overtaken government-led funding, which has moved the balance towards more applied research than basic research. This issue raises the question of who is reaping the benefits of research and if a "greater good" is achieved by it (Figure 6.4). $\{3.8\}$ For many measures there is a strong imbalance in data access between developed and developing countries, which contributes to global differences in countries' ability to understand the environment, the implications of this understanding for human health, and the use of environmental data for socioeconomic benefits $\{25.2 .2$; SPM $\}$
Figure 6.4: Equity questions in data and knowledge

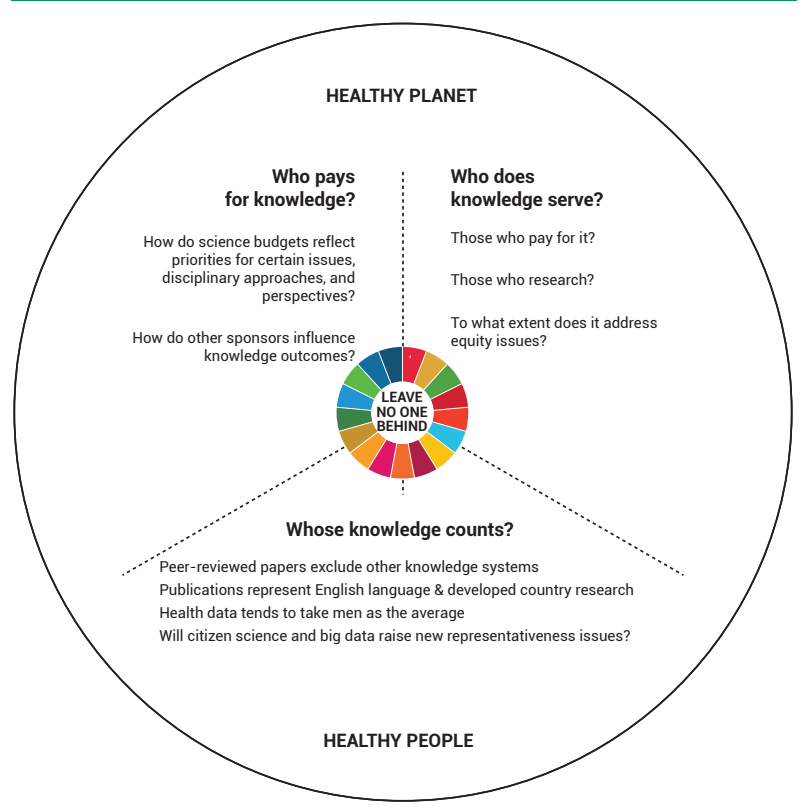

Source: UNEP (2019a, p. 66)

\subsection{The Need for Open Data and International Cooperation}

Currently much environmental data are collected as part of one-off studies or projects, limiting their usefulness (well established). In addition to filling knowledge gaps with new data, enormous gains can be made through consolidating, curating, harmonizing and increasing open access to existing data which might otherwise be widely dispersed and not easily be combined or compared (well established). Common frameworks, initiatives and political will are needed to merge data sources and make better use of what is available, and to adequately compensate for spatial differences in the ability to generate and assess data - as well as to undertake disaggregated information collection that reflects economic, health and equity issues. In this context, the Framework for the Development of Environment Statistics, the System of Environmental-Economic Accounting, and the System of National Accounts are robust consensusbased statistical frameworks and methodological approaches that could be broadly adopted (Figure 6.5). Rationalizing both existing and newly collected data is essential for the development of indicators. $\{3.3 ;$ SPM $\}$ 


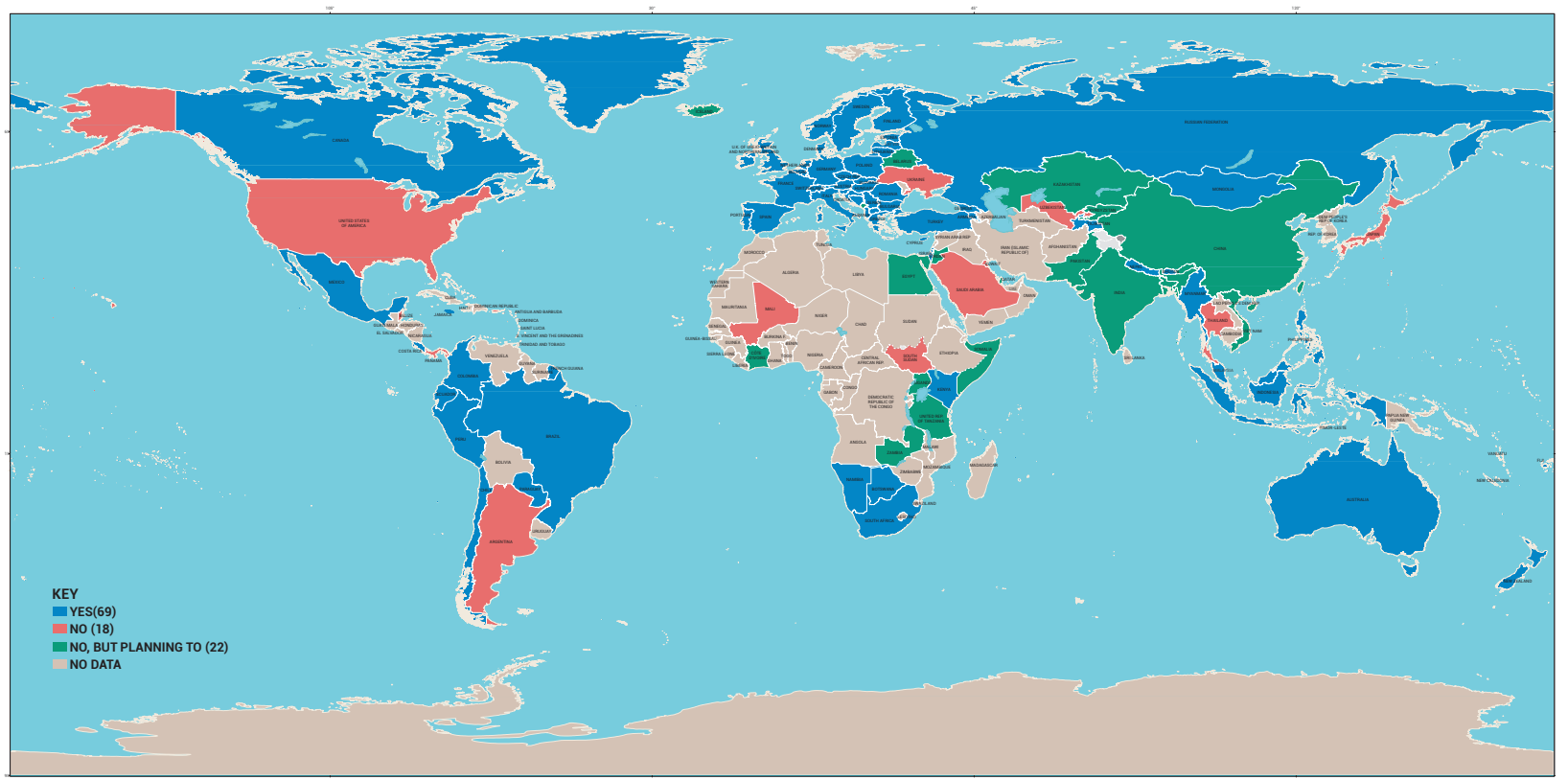

Source: United Nations (2018b).

Actions need to be taken on the basis of the knowledge already at hand, but the world needs openly accessible data, information, analysis, knowledge and science to better inform and guide what must be done to achieve sustainability across all environmental dimensions (established but incomplete). Achieving the SDGs, MEAs and other internationally agreed environmental goals and science-based targets will require an integrated approach that considers linkages across different environmental and non-environmental components, building upon disaggregated data generation and incorporating traditional knowledge and citizen science. The achievement of the SDGs and their targets needs to be followed up on and reviewed, using their global indicators and complemented by indicators at the national and regional levels. Work is also needed to develop the baselines for targets for which national and global baseline data do not yet exist. Integrated data and analysis can prioritize needs, shape effective policies, and strengthen monitoring and evaluation outcomes $\{3.1,25.1$; SPM, p. 23\}

\footnotetext{
More inclusive and open access to data will assist in achieving equity, transparency, and the best data use for sustainability and development (established but incomplete). The "open data" movement has gained significant traction in recent years, working towards data being freely available to all. Education is a key component of access. Countries should be forwardthinking in building the capacity to analyse and interpret environmental data. $\{25.4 .2\}$
}

International cooperation and sharing of data and information are key to success (well established). Continued investment in the education and training of the next generation of experts and decision makers is essential to maintain the pace of progress on the multigenerational challenges associated with the "Healthy Planet, Healthy People" theme. \{25.3; SPM\}. Sustainable capacity is achieved when the entire value chain works: when data from collation and analysis through to use are dealt with competently, in order to deliver the value of the information, disseminate it through the most effective and efficient channels, and ensure that those for whom its value is intended are able to use it. To reach this stage of competency, environmental education should be implemented at all levels. Indigenous knowledge systems, including emerging opportunities offered by technological advances to democratize the participation of citizens in the exercise of science and scientific discovery, should be facilitated. $\{25.4 .6\}$

\section{Data gaps will be an ongoing reality in the foreseeable future and should not delay urgent} action (well established). Decision makers at all levels cannot wait for new data before acting. Instead, they should implement evidence-based management based on current knowledge and be adaptive and responsive as new knowledge becomes available. Governments and society need to embrace the evolving data landscape, facilitate the development of 
new information technology skills, and adopt a holistic approach in utilizing both existing and emerging data and knowledge tools. \{25.2.4; SPM, p. 24\}

Transforming human-environment interactions (and related human-human interactions) towards sustainability, especially in the case of consumption and production patterns and lifestyles, requires a better information base as well as new, diversified knowledge of planetary systems and transformative processes within globalized social and economic systems. This includes the cultural dynamics and ethical foundation of human perceptions and understanding of "nature and environmental sustainability" and the need to incorporate people's health and well-being. $\{1.1\}$ 


\section{References}

Oki, T. and Kanae, S. (2006). Global hydrological cycles and world water resources. Science 313(5790), 1068-1072. https://doi.org/10.1126/ science. 1128845 .

United Nations (2018a). Tier Classification for Global SDG Indicators. https://unstats.un.org/sdgs/files/Tier\%20Classification\%20of\%20

SDG\%20Indicators_11\%20May\%202018_web.pdf.
United Nations (2018b). Global Assessment of Environmental-Economic Accounting and Supporting Statistics 2017. https://unstats.un.org/unsd/ statcom/49th-session/documents/BG-Item3h-2017-Global-Assessmentof-Environmental-Economic-Accounting-E.pdf.

United Nations Environment Programme (2019). Global Environment

Outlook - GEO-6: Healthy Planet, Healthy People. Nairobi.

https://wedocs.unep.org/bitstream/handle/20.500.11822/27539/

GEO6_2019.pdf? sequence=1\&isAllowed=y. 